\title{
Commentaries from Dissolution Tester Equipment Manufacturers on Enhanced Mechanical Calibration (MC) and Performance Verification Standards (PVT)
}

\author{
Vivian A. Gray \\ Dissolution Technologies, Hockessin, DE
}

Dor his Special Edition on Industry trends on MC and PVT would not be complete without the comments from the manufacturers of Apparatus 1 and 2. We asked for comments from the experienced and knowledgeable people in these companies. The comments below are listed in the order in which they were received. Some gave an assessment of trouble areas in the MC and PVT, whereas others reported on the trends they were seeing in the industry in the use of the MC and PVT.We appreciate this insightful feedback for the Special Edition.

\section{Royal Hanson}

Hanson Research Corporation

www.hansonresearch.com

In the thirty-five years that I have been involved with USP Apparatus 1 (basket) and Apparatus 2 (paddle), I have seen a steady refinement in the design and manufacture of these apparatus by the major vendors, to the extent that critical parameters and operation are now fairly well standardized. Equipment qualification procedures, however, may still be evolving.

Mechanical versus Chemical Calibration

This is one of the most contentious debates I have ever seen in our industry. On one hand, the USP specifies performance verification testing (PVT) of the dissolution test apparatus using "calibrator tablets." On the other hand, ASTM Standard E2503-07, which is supported by the FDA, specifies a mechanical calibration procedure that eliminates any further requirement for chemical (calibrator tablet) calibration. USP is generally recognized as the drug standards authority, whereas FDA is acknowledged as the enforcement agency. In my opinion, in this case, FDA has staked out competing and contradictory ground as the drug standards authority. The situation can be very confusing not only to vendors, but most importantly, to the pharmaceutical analyst. Which standard should be followed? In my judgment, mechanical calibration equates to $\mathrm{OQ}$ (operational qualification), and chemical calibration (PVT) equates to PQ (performance qualification). I believe both are ultimately of value in equipment qualification. However, my sense is that industry is leaning more and more towards ASTM mechanical calibration in lieu of USP PVT calibrator tablets.

\author{
Hans-Jurgen Knitter and JR Alicea \\ Erweka \\ www.erweka.com
}

First, the dissolution apparatus has to fulfill all the requirements according the mechanical calibration FDA Guidance for Industry, The Use of Mechanical Calibration of Dissolution Apparatus 1 and 2-Current Good Manufacturing Practice (CGMP), from January 2010. This is very important to minimize the hydrodynamic impact to the formulation. The dissolution medium has to be degassed according to USP Chapter $<711>$. Alternative validated methods that lead to the same result can also be used. The sampling has to be performed only at the specified time point. Additional sampling time points can lead to higher results.

The filtration of the sample has to be performed directly in the vessel (i.e., with an inline filter) at the correct position described in the USP as "midway between the surface of the Dissolution Medium and the top of the rotating basket or blade, not less than $1 \mathrm{~cm}$ from the vessel wall."

The filter has to be validated according to USP Chapter $<1092>$ The Dissolution Procedure: Development and Validation. Otherwise, the obtained data can be too low.

\section{John Burmicz \\ PHARMA TEST \\ www.pharma-test.com}

Despite all of the concerns voiced on several fronts, it seems that the new PVT has been a success. We have found that many of the tests have passed with the first set of six results. Depending on the age and state of the instrument, about $30 \%$ of tests have to be repeated for a second-stage analysis. In this case, most baths pass the test, and this, of course, depends on the service history of the bath, its regular maintenance, and the conditions under which the test is carried out. In general, the requirement for a minimum range of $\mathrm{CV}$ among vessels is not outrageous. The need to run each vessel with the medium correctly deaerated and with minimum tool distortion and vessel conformity is not an unreasonable requirement.

I am still of the firm belief that the argument for only the mechanical tests was embraced by people who thought that this was a panacea to somehow escape the 
six-month "trial" of the prednisone tests. While I agree that the statistical application of USP certification is somewhat confusing, the use of the calculation tool to minimize the required intellectual gymnastics has been a first-rate success. More importantly, mechanical testing alone is not a test of the interface between operator and instrument and the ability to follow and execute a simple SOP.There is no care required for the degree of deaeration, the environment in which the dissolution instrument is placed, and the attention with which the test is carried out. The extra degrees of instrumental restriction such as basket dimensions, run out, and so on are to be welcomed as a step forward in prescribing the universality of the dissolution test, but it is not a substitute for the test itself and the operator's ability to carry it out. This is the most important point. After all, tablets are tested with these instruments, not with dials and gauges.

To this end the new test is fine, and regardless of the age of instruments (I had two 18-year-old instruments pass the first time), there is every chance to pass this test as long as the bearings are not worn, high-frequency vibration is not transmitted down the tool shafts from worn belts, and the environmental vibration that occurs in a normal lab environment is minimized. It is up to the $\mathrm{OQ}$ team to get the metrology right. On the dissolution side, the initial temperature of the medium in each vessel, the tool wobble, and the technique needed to drop tablets and retrieve samples at the correct time interval means that this test is actually reasonable in its requirements and more of a true indication of the interface between man and machine.

\section{Bryan Crist}

Agilent Technologies, Inc.

www.agilent.com

Laboratories appear to be moving to enhanced Mechanical Calibration (MC), also referred to as Mechanical Qualification (MQ) in some standards, because of anticipated time and cost savings, the FDA's claim of a reduction of variability, and the perception that something is wrong with the traditional PVT. Our perception is that there are three relatively equal positions that dissolution laboratories have taken with regard to the traditional USP Performance Verification Test (PVT) and the enhanced Mechanical Calibration (MC) procedures from the ASTM and the FDA: (1) those who transitioned from the traditional PVT to enhanced MQ, (2) those who perform both PVT and enhanced MC in an investigational phase, and (3) those who continue to perform the traditional USP mechanical tolerances with PVT.

We observed that many makes and models of dissolution apparatus tuned within their enhanced mechanical qualification tolerances may still fail the new PVT acceptance criteria that focus more on variability. Two questions arise: Why does the PVT fail when the MC passes? Are laboratories performing MC for the right reasons?
Why Does the PVT Fail When the MC Passes?

It is true that prednisone is quite sensitive, yet from a review of numerous very tight sets of data with very low percent CV, excessive lot-to-lot variability of prednisone used in the PVT does not appear to exist. Some apparatus fail the PVT due to the sensitivity of USP prednisone when used with various apparatus components and configurations. The FDA guidance for industry on the subject of mechanical calibration of Apparatus 1 and 2 states,"that appropriate measures be taken to control the following sources of significant variability in dissolution testing: dissolved gases, vibration, and vessel dimensions" (1).

Looking at these three sources of significant variability, the MC alone cannot stand without the implementation of additional controls, procedures, or verifications to identify and eliminate analytical variability and bias from dissolved gasses (deaeration), vibration, and vessels. In addition, the condition of paddle and basket components as well as unvalidated automatic sampling equipment with resident sampling probes contribute to excessive variability.

\section{Are Laboratories Going to MC for the Right Reasons?}

Certain factors do arise which require maintenance and replacement to address mechanical issues. Failure to pass physical parameter specifications has been related to several key areas. Centering systems may be broken, missing, or malfunctioning. Some are replaced with thirdparty systems that do not function as well as original manufacturer systems. Although component dedication to an apparatus is required by MC procedures, some laboratories continue to interchange paddles, basket/ shafts, and vessels. Although dimensions are standard, there is no specific condition for wear; subjectivity leads to the use of components in poor condition. Vessel construction varies widely throughout the world resulting in vessels ranging from excellent to grossly misshapen. Across the globe, several sources of vessels with verticality, cylindrical, and deformity issues were noted. Manual gauges are very difficult to read within a vessel, and measurements can be subjective.

In summary, mechanical qualification may work on its own if additional systems are in place to eliminate the effects of deaeration, vibration, and vessel consistency. Additionally, paddle, basket/shaft, and vessel components must be individually verified and dedicated to specific apparatus. Certificates stating that paddles, basket/shafts, and vessels were manufactured to meet USP requirements are not Certificates of Conformance if they do not include individual measurements of each critical dimension outlined in the USP General Chapter $<711>$ Dissolution. In closing, some laboratories have stated that they switched to the enhanced MC because of unresolved failures associated with the new PVT acceptance limits. This is not always associated with physical parameter adjustments but usually a set of conditions that are not observed or considered. 


\section{Patrick Mahn}

Distek, Inc.

www.distekinc.com

First of all, we have not seen the huge move to ASTM that some of us expected once the FDA released its Guidance to Industry allowing the use of enhanced mechanical qualification in lieu of the USP traditional chemical qualification. There has been significant movement to the ASTM standard but not as much as one would probably have thought. The labs that have made the change have done so with great caution. Most get professional serialization of the dissolution parts and, to a much lesser degree, certification of all parts. The certification includes the physical measurements of paddles, baskets, and shafts. In our service business unit, we have seen about a $25-30 \%$ section of our customers moving to the ASTM standard. Please keep in mind that this is a rough estimate. It takes into consideration the number of baths and not necessarily the number of customers.

An interesting trend that this has produced is that a number of labs have actually tried to use both standards. They utilize the more strict specifications from each of the USP Toolkit and the ASTM standard. Then they run the Prednisone PVT tablets.

Regarding the performance of the tablets themselves, we have noticed a slight decrease in the percent dissolved for paddles. At this time last year, most paddles were getting geometric means (GM) of about 35-38. Today, we see more of a 32-36 range. The CVs continue to cause most of the headaches. Apparatus rarely fail GM, but the CVs continue to fail. Some of this is attributable to vessel shape and the inconsistencies from vessel to vessel. The basket tests have proved somewhat more stable with only occasional failures. Again, the culprit is the CV.

While some of our customers have observed failing CVs due to abnormally high or low results in the same position each test, most find that the outlier moves from position to position. Our team has found similar results.

We do the single-stage test for most of our customers that have us run these tests, while most customers that perform the tests themselves run two-stage tests. Quite often, they go to Stage 2 for paddles but not as often for baskets. If I had to make a guess, it would be that they fail Stage 1 about $50 \%$ of the time for paddles and $25 \%$ of the time for baskets.

\section{Michel Magnier and Marc Finn}

Sotax Corporation

www.sotax.com

\section{Trends in USP Apparatus 1 and 2 Qualification}

Observations in Europe and the United States obtained through our network of sales and service channels show that the majority of dissolution testing laboratories are continuing to use the PVT as the primary method for dissolution system validation. However, we have observed a dramatic interest in more rigorous mechanical calibration measurements because of the Mechanical Qualification described in ASTM E2503-7.

As laboratories gain more experience with and confidence in the new mechanical procedures, it is possible that a larger percentage of them will shift the emphasis more toward a mechanical qualification and eventually discontinue the use of the PVT. As an early indication of this, we have seen dissolution laboratories that perform six-month interval PVT qualifications switch to a twelve-month PVT schedule supplemented with six-month mechanical qualifications. We have also observed new system installations perform traditional IQ/OQ and PVT and then continue with only mechanical qualifications. SOTAX supports the PVT as an important part of a complete system qualification. The PVT is particularly useful for demonstrating equivalency between manual and automated sampling and analysis. SOTAX also supports the ASTM procedure as an improvement to the traditional USP OQ procedure. This technique can be used to identify failure investigations and to measure dissolution bath quality and longevity during its daily use.

\section{REFERENCES}

1. The Use of Mechanical Calibration of Dissolution Apparatus 1 and 2-Current Good Manufacturing Practice (CGMP); Guidance for Industry; U.S. Department of Health and Human Services, Food and Drug Administration, Center for Drug Evaluation and Research (CDER), U.S. Government Printing Office: Washington, DC, January 2010. 